\title{
Status of the OPERA experiment
}

\section{Francesco Di Capua}

Universita "Federico II" and INFN

E-mail: francesco.di.capua@cern.ch

The OPERA experiment has been designed to observe an unambiguous signal of $v_{\mu} \rightarrow v_{\tau}$ oscillation in the parameter region indicated by previous experiments in the atmospheric sector. It is based on the nuclear emulsion technique to directly observe among the neutrino interaction products the track of a $\tau$ lepton produced by a $v_{\tau}$ appeared in a $v_{\mu}$ beam. The OPERA detector is presently under construction in the Gran Sasso underground laboratory, $732 \mathrm{Km}$ away from CERN, where the CNGS $v_{\mu}$ beam will produce its first neutrinos in 2006. The experimental technique is described and the development of the project is reported. The physics potential in measuring $v_{\tau}$ appearance and in searching for $v_{e}$ appearance is discussed.

International Europhysics Conference on High Energy Physics

July 21st - 27th 2005

Lisboa, Portugal 


\section{The CNGS neutrino beam}

Following the discovery of neutrino oscillations by Super-Kamiokande in 1998 with atmospheric neutrinos [1], the CNGS long baseline project (CERN Neutrinos to Gran Sasso) is focused on the appearance of $v_{\tau}$ in a $v_{\mu}$ beam in order to have an unambiguous evidence for $v_{\mu} \rightarrow v_{\tau}$ nature of the atmospheric oscillation and check the $\Delta m^{2}$ value in this channel. Searching for the sub-leading $v_{\mu} \rightarrow v_{e}$ oscillations in this beam will also provide a window of opportunity to measure $\theta_{13}$ before the next generation of dedicated experiments.

The CNGS [2,3] is a wide band neutrino beam optimized for $\tau$ appearance with a mean neutrino energy of about $17 \mathrm{GeV}$, a $v_{e}$ and $\bar{v}_{e}$ contamination of $0.87 \%$ and a negligible prompt $v_{\tau}$ contamination. The yearly event rate in the OPERA detector correspond to $6200 v_{\mu}$ charged and neutral current interactions, while $27 v_{\tau}$ charged current interactions are expected per year for a $\Delta m_{23}^{2}$ of $2.4 \times 10^{-3} \mathrm{eV}^{2}$. The present time schedule foresees a hardware test at the beginning of january 2006 and the commissioning will start in may 2006. The first neutrino beam useful for physics will be delivered at the Gran Sasso in July 2006.

\section{The OPERA experiment}

\subsection{The detector principle and its structure}

The principle of the OPERA experiment is to observe the $\tau$ short track and the decay products by means of thin emulsion layers. To provide the large target mass (1.8 ktons) the emulsion films are interleaved with $1 \mathrm{~mm}$ thick lead plates. An emulsion film in OPERA consists of two emulsion layers ( $45 \mu \mathrm{m}$ thick) on either side of a plastic base (200 $\mu \mathrm{m}$ thick). The basic detector unit, called ECC (Emulsion Cloud Chamber) brick, is obtained by stacking 56 lead plates and 57 emulsion films, plus two additional emulsion films, called Changeable Sheet (CS), behind $2 \mathrm{~mm}$ of plastic. The CS's can be detached from the rest of the brick for analysis. They will be used to locate the tracks produced in neutrino interactions to be followed in the rest of the brick. The angular resolution of about $2 \mathrm{mrad}$ and a space resolution better than 1 micron, allow for the vertex and kink topology reconstruction. The dimensions of a brick are $12.7 \times 10.2 \times 7.5 \mathrm{~cm}^{3}$ and its mass about $9 \mathrm{~kg}$. In terms of radiation lengths, a brick corresponds to a thickness of $10 \mathrm{X}_{0}$. The brick is a completely standalone detector allowing not only for the event topology reconstruction but also for: a) the measurement of the momenta of charged particles produced in the interactions by looking at their multiple scattering in the lead plates when comparing track segments in successive emulsion sheets; b) the identification of electrons and photons and the measurement of their energy by sampling electromagnetic shower with emulsion sheets; c) the pion to muon separation by looking at the $d E / d x$ in the emulsions, measured close to the track end of range. In order to reach a 1.8 kton mass, about 206000 bricks will be installed into walls each containing 64 rows of 52 bricks and equipped with planes of electronic trackers. The ECC bricks are assembled by means of an automatic machine (BAM).

Fig. 1 shows the general layout of the OPERA detector. It consists of 2 identical parts called Super Modules (SM). Each SM consists of a target section and a muon spectrometer. The spectrometer consists of $5 \mathrm{~cm}$ thick iron slabs $10 \mathrm{~m}$ high and $9 \mathrm{~m}$ wide and it weights about 1000 tons. 
The spectrometer is equipped with drift tubes, that provide high precision tracking, and RPC chambers (each magnet has an equivalent RPC surface of $1540 \mathrm{~m}^{2}$ ). These systems measure the charge and the momentum of through going muons by means of a dipolar magnet (1.6 Tesla transverse to neutrino beam). The momentum resolution for $20 \mathrm{GeV}$ muons is about $25 \%$. The target section is composed of 31 walls of bricks. The brick support is designed to insert or extract bricks from the sides of the walls by using an automated manipulator (BMS). An electronic target tracker module, installed behind each brick wall, consists of a plane of $6.9 \mathrm{~m}$ long scintillator strips in each transverse directions ( $\mathrm{X}$ and $\mathrm{Y}$ ). The strips, $2.6 \mathrm{~cm}$ wide and $1 \mathrm{~cm}$ thick, have a WLS fibers for readout by 64 channel multi-anode photomultiplier tubes. The transverse pointing accuracy is about $1.5 \mathrm{~cm}$ for $\mathrm{CC}$ events and $3.0 \mathrm{~cm}$ for NC events. The main goal of the electronic detectors is to provide a trigger for the neutrino interactions and to localize the brick where the event occurred. The brick selected as the one in which the neutrino interacted is hence extracted from the target and its emulsion sheets are scanned with automatic microscopes.

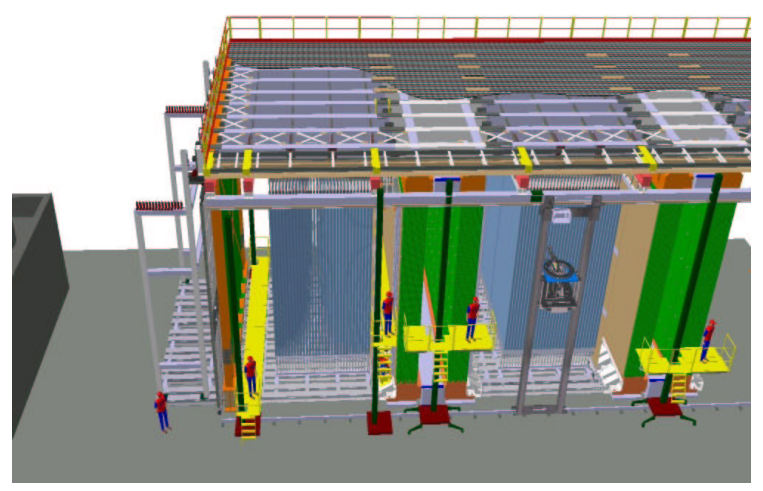

Figure 1: Layout of the OPERA detector with two supermodules.

\subsection{Construction status at the LNGS}

The installation of the OPERA experiment in Hall C of the Gran Sasso underground laboratory started in May 2003 with the construction of the spectrometers. The construction and the cabling of both spectrometers (iron slabs plus RPC) as well as of the overall mechanical structure has been completed in September 2005. A test of four RPC planes, interleaved with iron slabs but without magnetic field, has been performed. A rate of $15.6 \pm 0.5 \mu$ /hour has been measured and it is in very good agreement with the value of $15.2 \pm 0.3 \mu$ /hour estimated by using the MACRO Monte Carlo. The spectrometer of the first SM has been also equipped with the drift tubes. The installation of the brick walls and target tracker planes in SM1 is in progress and so far 26 out 31 planes have been mounted. By using the final DAQ of the experiment the muon rate has been measured by using 6 TT planes and it turned out to be $18 \mu$ /hour. The completion (with walls filled with ECC bricks) of the first SM is expected by the end of July 2006, in time for the observation of the first neutrinos coming from CERN. Given the high modularity of the OPERA target, the filling of the second SM with bricks may proceed without interference with the normal data taking of the first spectrometer. About $80 \%$ of the nuclear emulsions has been produced by the Fuji film company and half of them 
already refreshed (i.e. the tracks collected during the production are removed through a special procedure) at the Tono Mine (Japan) and shipped at Gran Sasso.

\subsection{Background and detection efficiency studies}

The main background sources for $v_{\mu} \rightarrow v_{\tau}$ oscillation search are charmed decays, hadron reinteractions and large angle muon scattering.

The charm production induced by neutrinos is a background source when the primary muon is not identified (typically it happen for low energy muons). By using a combined analysis of the electronic detectors and of the ECC bricks, it is possible to achieve an efficiency in the muon identification of about $95 \%$. However, there is another handle to identify low momentum muon and, consequently, reduce the charm background. It exploits the different energy loss, due to a different mass, of pions and muons at the end of their range. ECC exposures have been performed in the pure and low energy muon and pion beams available at the PSI. The preliminary results are encouraging: about $30 \% \mu \mathrm{ID}$ efficiency with a few $\% \pi$ mis-identification.

The prong multiplicity and angular distribution in pion interactions on lead nuclei below $10 \mathrm{GeV}$ are poorly known. However, this process represents the main source of background in the hadronic channel. In order to accurately measure this background, exposures of a brick to pion beams from 1 to $6 \mathrm{GeV}$ have been carried out and the analysis is in progress (foreseen precision $\sim 10 \%$ ). We also plan to measure this background in the OPERA experiment itself. Indeed, once the primary neutrino interactions is reconstructed, it will be possible to search for decay-like topologies outside the fiducial volume used to search for $\tau$ decays. This will give a direct, Monte Carlo independent, measurement of the expected background in the $\tau$ candidate sample.

Presently, the main background for the muonic channel is the large angle scattering of muons off $1 \mathrm{~mm}$ lead. There are very scarce data about the rate of this process. On the other hand there is about one order of magnitude discrepancy between the predicted rate with GEANT3 and an analytical calculation [4] that gives a lower rate (background). An exposure with a silicon tracker telescope and a pure $\mu$ beam has been performed. The analysis is still in progress, but the expected background measured with an empty target is of the order of $10^{-6}$ and it will allow the measurement with the needed accuracy.

In order to validate the Monte Carlo evaluation of the detection efficiencies, we plan to search for all charm decay topologies (both neutral and charged) when the primary muon is correctly identified and attached to the primary vertex. The expected number of charm produced in the target can be extrapolated from the CHORUS measurementsn with an accuracy in the 10-20\% range. Therefore, the decay detection efficiency for each decay topology can be checked with such an accuracy.

All the measurements discussed above will provide solid bases for the OPERA results.

\section{$2.4 v_{\mu} \rightarrow v_{\tau}$ sensitivity}

The $\tau$ candidate events are selected on the basis of the existence of a kink or of a multiprong decay topology along the parent track. In order to improve the signal to noise ratio, after the topological selection of the candidates a kinematical analysis is applied. The details of the performances of the kinematical variables reconstruction as well as the efficiency of the kinematical 
analysis may be found in Refs. [4, 5]. The detected number of $\tau$ events and the corresponding background after 5 years of data taking with the nominal CNGS intensity, as a function of $\Delta m^{2}$ and for full mixing, are shown in Table 1. The second row of Table 1 gives the expected background after the foreseen improvements both on the large angle muon scattering and muon identification in emulsions are achieved.

Table 1: Summary of the OPERA signal and background for different values of $\Delta m^{2}$ at full mixing.

\begin{tabular}{lllll}
\hline & $1.910^{-3} \mathrm{eV}^{2}$ & $2.410^{-3} \mathrm{eV}^{2}$ & $3.010^{-3} \mathrm{eV}^{2}$ & Back. \\
\hline & 8.0 & 12.8 & 19.9 & 1.0 \\
tback.reduction & 8.0 & 12.8 & 19.9 & 0.8 \\
\hline
\end{tabular}

The discovery potential of the experiment is shown in Fig. 2. It gives the probability of observing in 5 years a number of events larger than a background fluctuation with 4 gaussian $\sigma$ equivalent probability. The continuous and the dashed lines correspond to the first and the second row of Table 1, respectively. The latest $90 \%$ C.L. Super-Kamiokande allowed region based on the $\mathrm{L} / \mathrm{E}$ analysis is reported for comparison. From this plot one may conclude that in the worst case (lowest allowed $\Delta m^{2}$ ) there is a $\sim 70 \%$ probability to observe $v_{\mu} \rightarrow v_{\tau}$ oscillations with a $4 \sigma$ significance. In case no signal is observed, the OPERA experiment will be able to exclude the $99 \%$ C.L. Super-Kamiokande plus K2K allowed region with a C.L. of 99\%, see Fig. 3.

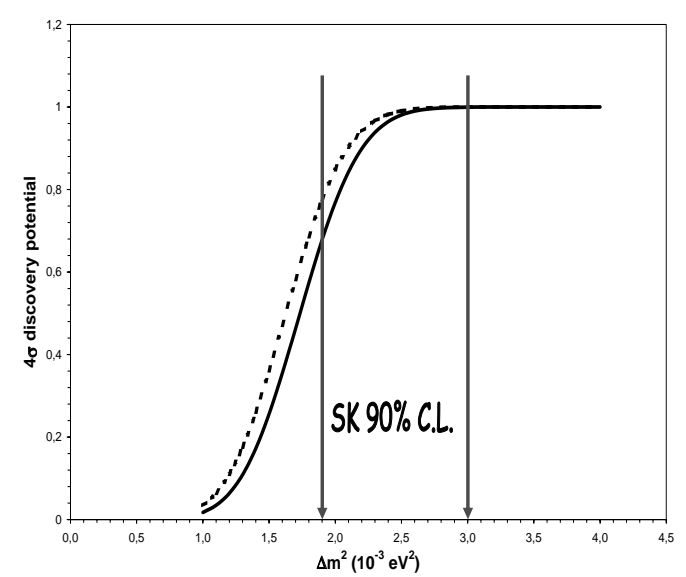

Figure 2: OPERA discovery potential.

\section{$2.5 v_{\mu} \rightarrow v_{e}$ oscillation search}

Given the good electron identification capabilities in the bricks, OPERA can look as well for $v_{\mu} \rightarrow v_{e}$ oscillations. For $\theta_{13}=9^{\circ}$ and $\Delta m_{23}^{2}=2.5 \times 10^{-3}$, after 5 years of data taking, 9.3 signal events are expected. The main backgrounds are: the intrinsic $v_{e}$ beam contamination (18 events); $\pi^{0}$ identified as electron in $v_{\mu}$ neutral current events (5.2 events) or charged current events with the muon not identified (1.0 events); electrons coming from $\tau$ decays in $v_{\mu} \rightarrow v_{\tau}$ oscillations (4.5 


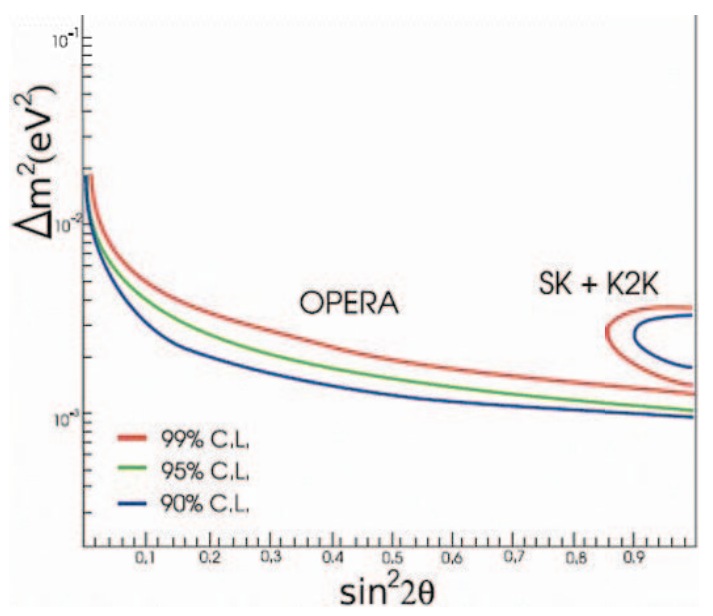

Figure 3: OPERA exclusion plot computed at $99 \%, 95 \%$ and $90 \%$ C.L.

events). The signal to background ratio can be enhanced by performing a simultaneous fit to the distribution of the visible energy, electron energy and missing transverse momentum.

After 5 years data taking, although not optimized for $v_{\mu} \rightarrow v_{e}$, the OPERA experiments may improve, depending on the $\delta_{C P}$ value and matter effects, the present CHOOZ limit by a factor $\sim 1.5$ $[6,7]$.

\section{References}

[1] Y. Fukuda et al. [Super-Kamiokande Collaboration], Phys. Rev. Lett. 81 (1998) 1562 [arXiv:hep-ex/9807003].

[2] G. Acquistapace et al., CERN-98-02

R. Baldy et al., CERN-SL-99-034-DI

[3] Home page of the CNGS project http://proj-cngs.web.cern.ch/proj-cngs)

[4] M. Guler et al. [OPERA Collaboration], CERN-SPSC-2000-028

[5] P. Migliozzi, Int. J. Mod. Phys. A 18 (2003) 3877.

[6] M. Komatsu, P. Migliozzi and F. Terranova, J. Phys. G 29 (2003) 443 [arXiv:hep-ph/0210043].

[7] P. Migliozzi and F. Terranova, Phys. Lett. B 563 (2003) 73 [arXiv:hep-ph/0302274]. 